\title{
The critical sensor: a new type of evanescent wave immunosensor
}

\author{
E.F. Schipper, R.P.H. Kooyman, A. Borreman \& J. Greve \\ MESA Research Institute, Department of Applied Physics, Bio-Interface Group, University of Twente, \\ P.O. Box 217, 7500 AE Enschede, The Netherlands \\ Tel: [31] (53) 893112 Fax: [31] (53) 4891105
}

(Received 27 January 1995; revised 7 June 1995; accepted 12 June 1995)

\begin{abstract}
A new planar waveguide immunosensor has been developed in which adsorption at a surface, changing the refractive index contrast, is measured. In this "critical" sensor the change in the effective refractive index contrast is transducted to a shift of the critical reflection angle. The sensor's response after a specific binding of antigens to antibodies is discussed theoretically. In addition, an experimental sensitivity evaluation on the basis of several immunosensing experiments is presented. The obtained lower detection limit is $2 \times 10^{-2} \mathrm{~nm}$ in adlayer growth, equivalent to $12 \mathrm{pg} / \mathrm{mm}^{2}$ of analyte coverage. This sensitivity is comparable to the performance of the surface plasmon resonance sensors or the grating coupler sensors. However, the "critical" sensor has some advantages. These are mainly the ease of fabrication and adjustment prior to a measurement, and the fact that for an experiment no metal layer has to be used.
\end{abstract}

Keywords: immunosensor, planar waveguides, deflection, critical angle, evanescent wave sensor.

\section{INTRODUCTION}

In recent years the development of chemo-optical sensors has become a very active research area in university and industry. These sensors are intended to measure quantitatively and selectively low concentrations of analyte molecules. An interesting application can be found in the field of environmental monitoring. For example, the evaluation of the contamination of drinking water by traces of pesticides is of growing concern. in the European Union very low maximum concentrations are permitted, which can only be detected with laborious and time consuming techniques such as HPLC, gas chromatography, etc. Therefore, there is a growing need for fast and cheap sensor systems.

Optical evanescent wave sensors show a high optical surface sensitivity. Used as immunosensors these devices permit the detection of very low concentrations of antigens that selectively bind to antibody receptor molecules. The success of these sensors has been confirmed by the recent commercial development of some of them (Kooyman et al., 1991; Cush et al., 1993; Liedberg et al., 1993; Nellen \& Lukosz, 1993).

The immunoreaction between an antigen and an antibody immobilized on the surface of a metal or a dielectric layer induces a layer growth that changes the refractive index in the immediate vicinity of this surface. The plasmon or waveguide 
modes supported by the metal, or the dielectric layer, respectively, probe these optical changes within a few hundreds of nanometers from the surface. A variety of optical methods have been developed to read out these optical changes (Kooyman \& Lechuga, 1995).

The feasibility of optical waveguide sensors based on an interferometric detection principle has been demonstrated earlier by our group (Heideman et al., 1993). However, despite the very high intrinsic sensitivity of these interferometric sensors, there are some drawbacks: the optical alignment prior to a measurement can be time-consuming, and owing to the intrinsic periodic response an immunoresponse has always to be monitored in real time. The new simpler "critical" sensor overcomes these difficulties. A short account in which this "critical" sensor is compared with the Mach-Zehnder sensor has been published earlier (Schipper et al., 1995). Here, the principle and the prospects of the "critical" sensor will be discussed in more detail.

\section{THE DEVICE}

\section{Principle}

The principle of the "critical" sensor is based on the well-known Snellius law of refraction describing the deflection of light passing an interface between two media with different refractive indices:

$$
n_{1} \sin \alpha_{1}=n_{2} \sin \alpha_{2}
$$

where $n_{1}$ and $n_{2}$ are the refractive indices of the media 1 and 2, respectively; $\alpha_{1}$ and $\alpha_{2}$ are the corresponding angles of the light beams to the normal of the interface.

If the refractive index changes within the penetration depth of the evanescent volume of a guided lightmode, for example, when a protein layer is adsorbed on a waveguide surface, then the effective refractive index $\left(N_{\text {eff }}\right)$ will change (Lukosz, 1991). In this situation equation (1) holds equally well, with $n$ replaced by $N_{\text {eff }}$. An adsorbed layer can only change $N_{\text {eff }}$ if its refractive index is different from that of the medium. Part of the waveguide surface can be shielded, leaving $N_{\text {eff }}$ in this area constant. Then, after an adsorption the $N_{\text {eff }}$ contrast between a shielded and unshielded area will change, thereby changing the deflection angle of light passing the interface between the areas, as is represented in Fig. 1. This change, as a function of the layer growth, can be expressed by

$$
\Delta \theta=\int_{t_{\mathrm{s}}}^{t_{\mathrm{e}}} \frac{\mathrm{d} \theta}{\mathrm{d} N_{\mathrm{eff} 2}} \cdot \frac{\mathrm{d} N_{\mathrm{eff} 2}}{\mathrm{~d} t} \mathrm{~d} t
$$

where $\theta$ is the angle between the normal of the interface and the beam leaving this interface, $N_{\text {eff2 }}$ is the effective refractive index in the unshielded area, $t$ is the adlayer thickness, and $t_{\mathrm{s}}$ and $t_{\mathrm{e}}$ are the thicknesses of this layer on the waveguide before and after an adsorption, respectively.

The change in $N_{\text {eff } 2}$ caused by an adsorption can be measured as a change in the position of the deflected beam, as is done in the "normal" deflection sensor. A disadvantage of such a beam position measurement is the importance of the starting position of the beam. As a result, a calibration measurement before every new adsorption experiment is necessary.

In an alternative configuration, called the "critical" sensor, such a calibration measurement is less important. The critical angle for a reflection at an interface between two areas with different refractive indices is that angle above which light is totally internally reflected. This critical angle is a function of the $N_{\text {eff }}$ contrast at the interface:

$$
\theta_{c}=\arcsin \left(\frac{N_{\text {eff } 2}}{N_{\text {eff1 }}}\right) \quad \text { with } N_{\text {eff } 2}<N_{\text {eff } 1}
$$

The critical angle becomes a function of an adsorption if, similarly to the earlier discussed deflection configuration, a waveguide is partly shielded. Before an adsorption the direction of a divergent light beam, striking the interface between a shielded and unshielded area, can be tuned in a way that half of the light is reflected $(R)$ and half of it is transmitted (T), as can be

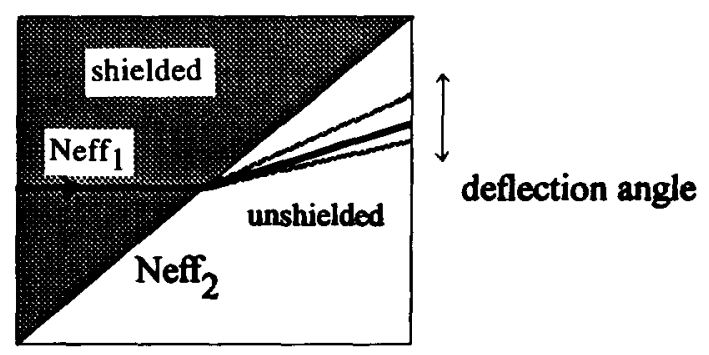

Fig. 1. The deflection effect at the sensor interface. 
seen in Fig. 2. A change in the critical angle due to an adsorption then results in a change of the difference between the $R$ and $T$ part:

$\Delta(R-T)=\int_{t_{\mathrm{s}}}^{t^{\mathrm{e}}} \frac{\mathrm{d}(R-T)}{\mathrm{d} \theta_{\mathrm{c}}} \cdot \frac{\mathrm{d} \theta_{\mathrm{c}}}{\mathrm{d} N_{\mathrm{eff} 2}} \cdot \frac{\mathrm{d} N_{\mathrm{eff} 2}}{\mathrm{~d} t} \mathrm{~d} t$

Here, $R$ is the intensity of the reflected and $T$ that of the transmitted part of the beam, respectively; $\theta_{\mathrm{c}}$ is the critical angle; $N_{\text {eff2 }}$ and $t$ are as defined before.

In contrast to the deflection sensor, with the "critical" sensor only an intensity difference has to be measured during an adsorption process. This offers a possibility for the development of a simpler sensor. The design of such a sensor optimized for the detection of a layer growth will be described in the following section.

\section{Sensitivity to layer growth}

For a direct monitoring of an adsorption caused by, for example, an immunoreaction we need a sensor with a high intrinsic sensitivity. To obtain this, the condition given by equation (4) has to be optimized. The first factor that can be optimized is $\mathrm{d} N_{\text {eff }} / \mathrm{d} t$. It can be expressed by (Lukosz, 1988):

$$
\begin{aligned}
& \frac{\mathrm{d} N_{\text {eff }}}{\mathrm{d} t}=\frac{\left(n_{\mathrm{f}}^{2}-N_{\mathrm{eff} 2}^{2}\right)}{\left(N_{\mathrm{eff} 2} \cdot t_{\mathrm{eff}}\right)} \cdot \frac{\left(n_{\mathrm{m}}^{\prime 2}-n_{\mathrm{m}}^{2}\right)}{\left(n_{f}^{2}-n_{\mathrm{m}}^{2}\right)} . \\
& \left\{\frac{\left(N_{\mathrm{eff}} / n_{\mathrm{m}}\right)^{2}+\left(N_{\mathrm{eff2}} / n_{m}^{\prime}\right)^{2}-1}{\left(N_{\mathrm{eff} 2} / n_{\mathrm{m}}\right)^{2}+\left(N_{\mathrm{eff} 2} / n_{\mathrm{f}}\right)^{2}-1}\right\}^{p}
\end{aligned}
$$

Here, $n_{\mathrm{f}}$ is the refractive index of the waveguide layer, $n_{\mathrm{m}}$ that of the medium, (typically $\left.n_{\mathrm{m}}=1 \cdot 34\right), n_{\mathrm{m}}^{\prime}$ that of the analyte molecules (typically $n_{m}^{\prime}=1.45$ ), $p$ is 0 for TE and 1 for TM polarization, and $t_{\text {eff }}$ is the effective waveguide layer thickness ( $=$ the waveguide layer thickness + the penetration depth of the light).

$\mathrm{d} N_{\text {eff }} / \mathrm{d} t$ is a function of $n_{\mathrm{f}}$ and the thickness

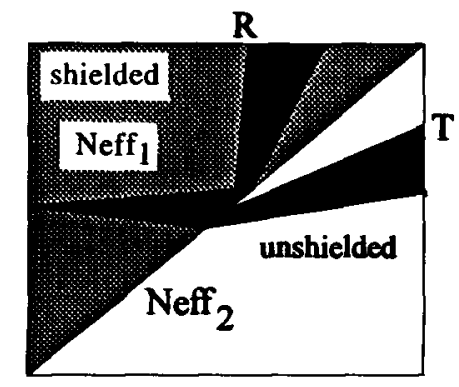

Fig. 2. The critical sensor measurement. of this layer. For different values of $n_{\mathrm{f}}$ between 1.6 and $2 \cdot 2$, the maximum obtainable value of $\mathrm{d} N_{\text {eff } 2} / \mathrm{d} t$ has been calculated for TE and TM polarized light. These values and the corresponding thicknesses are presented in Table 1. It can be concluded from this table that the maximum sensitivity is found at the highest refractive index contrast between the waveguide layer and the surroundings. However, an increase of the index contrast decreases the optimal thickness of the waveguide layer; $\mathrm{Si}_{3} \mathrm{~N}_{4}$ waveguide layers, which have a high refractive index $(n=2 \cdot 0)$, deposited on $\mathrm{SiO}_{2}$ substrate layers, fulfil these conditions and have proved to be suitable for sensor applications (Heideman et al., 1993).

The second factor that can be optimized in equation (4) is $\mathrm{d}(R-T) / \mathrm{d} \theta_{\mathrm{c}}$. To find a mathematical expression for this factor the shape of the intensity profile of the waveguide mode is required. The intensity distribution of a zerothorder slab waveguide mode in the plane of the waveguide can be considered Gaussian:

$$
g(\theta)=\exp -2\left(\frac{\theta}{\theta_{w}}\right)^{2}
$$

and:

$$
P=\int_{-\frac{1}{2} \pi}^{\frac{1}{2} \pi} I_{0} g(\theta) \mathrm{d} \theta
$$

Here, $g(\theta)$ is the intensity distribution function, $I_{0}$ is the intensity, $P$ is the laser power, and $\theta_{w}$ is the beam divergence.

The beam will split at the sensor interface into $R$ and $T$ parts. The $R$ part can be written as

TABLE 1 Maximum value of $\partial N_{\mathrm{efe} 2} / \partial t$ and the corresponding layer thickness, at different values of $n_{\mathrm{f}}\left(\lambda=632.8 \mathrm{~nm}, n_{\mathrm{s}}=1.46, n_{\mathrm{c}}=1.33, n^{\prime}=1.45\right)$.

\begin{tabular}{lccc}
\hline$n_{\mathrm{f}}$ & Mode & $\begin{array}{c}\text { Optimal waveguide } \\
\text { layer thickness } \\
\text { [nm] }\end{array}$ & $\begin{array}{c}\text { Maximum } \\
\text { sensitivity, } \mathrm{d}_{\text {eff2 }} \\
\mathrm{d} t\left(\mathrm{~nm}^{-1}\right)\end{array}$ \\
\hline 1.6 & & & $0.8 \times 10^{-4}$ \\
& $\mathrm{TE}_{0}$ & 130 & $0.9 \times 10^{-4}$ \\
1.8 & $\mathrm{TM}_{0}$ & 160 & $2.5 \times 10^{-4}$ \\
& $\mathrm{TE}_{0}$ & 80 & $2.9 \times 10^{-4}$ \\
& $\mathrm{TM}_{0}$ & 140 & $3.5 \times 10^{-4}$ \\
2.0 & $\mathrm{TE}_{0}$ & 60 & $4.3 \times 10^{-4}$ \\
& $\mathrm{TM}_{0}$ & 130 & $5.3 \times 10^{-4}$ \\
2.2 & $\mathrm{TE}_{0}$ & 50 & $5.6 \times 10^{-4}$ \\
\hline
\end{tabular}




$$
R=I_{0} \int_{-\frac{1}{2} \pi+\theta_{0}}^{\frac{1}{2} \pi+\theta_{0}} r(\theta)^{2} \exp -2\left(\frac{\left(\theta-\theta_{0}\right)^{2}}{\theta_{w}^{2}}\right) \mathrm{d} \theta
$$

Here, $\theta_{0}$ is the central incident angle of the beam to the interface, and $r(\theta)$ is the reflection coefficient at this interface.

Under the assumption that light absorption is absent, the intensity difference between the $R$ and $T$ parts can be written as

$$
R-T=2 R-P
$$

which results with equation (7) in

$$
\begin{aligned}
& R-T=2 I_{0} \int_{-\underline{z} \pi+\theta_{0}}^{\frac{3}{2} \pi+\theta_{0}} r(\theta)^{2} \exp -2\left(\frac{\left(\theta-\theta_{0}\right)^{2}}{\theta_{w}^{2}}\right) \mathrm{d} \theta-I_{0} . \\
& \int_{-\frac{1}{2} \pi+\theta}^{2 \pi+\theta_{0}} \exp -2\left(\frac{\left(\theta-\theta_{0}\right)^{2}}{\theta_{w}^{2}}\right) \mathrm{d} \theta
\end{aligned}
$$

During an adsorption on the waveguide surface the difference between the $R$ and $T$ parts changes, due to a change in the critical angle (see Fig. 2). In this figure the dark area is related to $R$, and the "white" area to $T$. When the critical angle increases, then $T$ increases as $R$ decreases. Using the fact that the reflection coefficient $r=1$ for $\theta>\theta_{c}$, we can write:

$$
\begin{aligned}
& \frac{\mathrm{d}(R-T)}{\mathrm{d} \theta_{\mathrm{c}}}=2 I_{0} \frac{\mathrm{d}}{\mathrm{d} \theta_{\mathrm{c}}} \int_{-\frac{1}{2} \pi+\theta_{0}}^{\theta_{\mathrm{c}}} . \\
& r(\theta)^{2} \exp -2\left(\frac{\left(\theta-\theta_{0}\right)^{2}}{\theta_{w}^{2}}\right) \mathrm{d} \theta+2 I_{0} \frac{\mathrm{d}}{\mathrm{d} \theta_{\mathrm{c}}} . \\
& \int_{\theta_{\mathrm{c}}}^{1 \pi+\theta_{0}} \exp -2\left(\frac{\left(\theta-\theta_{0}\right)^{2}}{\theta_{w}^{2}}\right) \mathrm{d} \theta
\end{aligned}
$$

When the beam divergence is high, it is possible to simplify this equation. If $\theta_{w}>0.14 \mathrm{rad}$, then the first component of equation (10) is less than $10 \%$ of the second component. In this situation we can write:

$$
\begin{aligned}
\frac{\mathrm{d}(R-T)}{\mathrm{d} \theta_{c}} & \leq 2 I_{0} \frac{\mathrm{d}}{\mathrm{d} \theta_{c}} \int_{\theta_{\mathrm{c}}}^{\frac{1}{3} \pi+\theta_{0}} \exp -2\left(\frac{\left(\theta-\theta_{0}\right)}{\theta_{w}^{2}}\right)^{2} \\
& \leq-2 I_{0} \exp -2\left(\frac{\left(\theta_{\mathrm{c}}-\theta_{0}\right)^{2}}{\theta_{w}^{2}}\right)
\end{aligned}
$$

It immediately follows from this equation that the maximum sensitivity $\mathrm{d}(R-T) / \mathrm{d} \theta_{\mathrm{c}}$ is obtained at $\theta_{0}=\theta_{c}$, which is of course not so surprising. Another aspect, not so apparent from equation (11) is illustrated in Fig. 3, which explains the difference in sensitivity when the divergence of the probing light beam is changed. Here, the intensity distribution is shown of a Gaussian beam with a low divergence (situation $\mathrm{A}$ ), and a Gaussian beam with a high divergence (situation B). The dark area below the Gaussian beam profile is a measure for $R$, whereas the combined grey and "white" areas are a measure for $T$. The grey portion of the total transmitted intensity in Fig. 3 denotes the incremental increase in $T$ when $\theta_{c}$ shifts, indicated with situation (1) to (2) to (3). It is obvious from this figure that, for a low divergent beam at $\theta_{\mathrm{c}} \neq \theta_{0}$, situation $\mathrm{A} 2$, the relative change in $R / T$ is much larger than for situation B2, where the beam has a larger divergence.

$\mathrm{d} \theta_{\mathrm{c}} / \mathrm{d} N_{\text {eff } 2}$ is the last factor of equation (4) which determines the sensitivity. This factor can be derived from the Snellius law:

$$
\frac{\mathrm{d} \theta_{\mathrm{c}}}{\mathrm{d} N_{\text {eff2 }}}=\frac{1}{\sqrt{N_{\text {eff } 1}{ }^{2}-\mathrm{d} N_{\text {eff } 2}{ }^{2}}}
$$

It follows from equation (10) that a maximum sensitivity $\mathrm{d} \theta_{\mathrm{c}} / \mathrm{d} N_{\text {eff2 }}$ is found when $N_{\text {eff2 }} N_{\text {eff1 }}$ ). In this situation, however, the critical angle increases to an impractical high value.

For constant laser power $P$ the sensitivity is influenced via $I_{0}$ by the beam divergence $\theta_{w}$ (see also Fig. 3). A highly collimated beam has a higher intensity $I_{0}$ than a divergent beam, and, therefore, the obtained sensitivity with this beam is higher. This is also demonstrated in Fig. 4, in which the change in the difference signal $(R-T)$, normalized by the intensity $(R+T)$, is calculated for different values of $\theta_{w}$ as a function of a change in $N_{\text {eff2 }}$ (for details see the captions of Fig. 4). A $10 \mathrm{~nm}$ protein layer adsorption is comparable to a change in $\Delta N_{\text {eff } 2}$ of $5 \times 10^{-3}$. The linearity of the $(R-T)$ change and the measurement range are also influenced by the beam divergence. We see from Fig. 4 that this linearity is worst for the beam with the lowest divergence. Simultaneously, the measurement range is relatively small for this situation, as can be concluded from Fig. 3 .

\section{MATERIALS AND METHODS}

\section{Waveguide structure}

The planar waveguides are fabricated using standard Si techniques. For an overview of the 


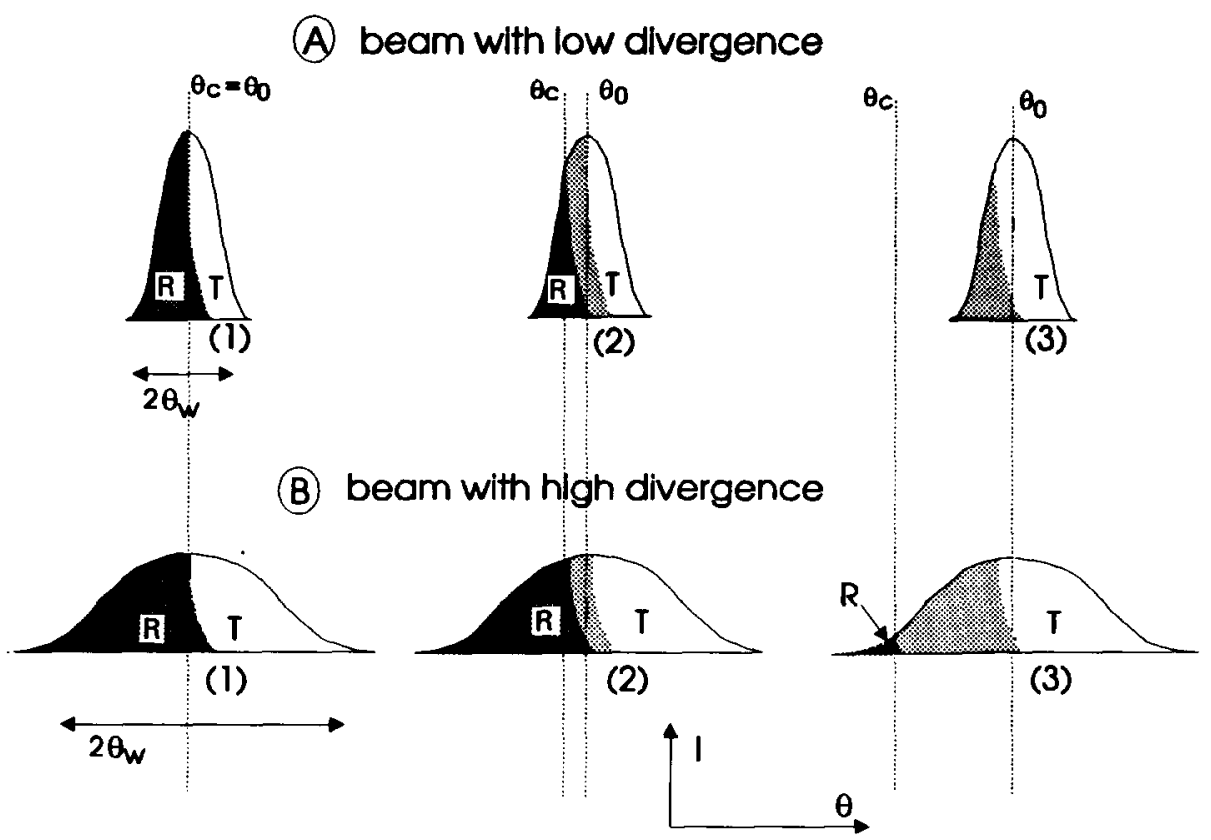

Fig. 3. The sensitivity difference between a Gaussian beam with a low divergence (situation A) and a Gaussian beam with a high divergence (situation B) (for details see text).

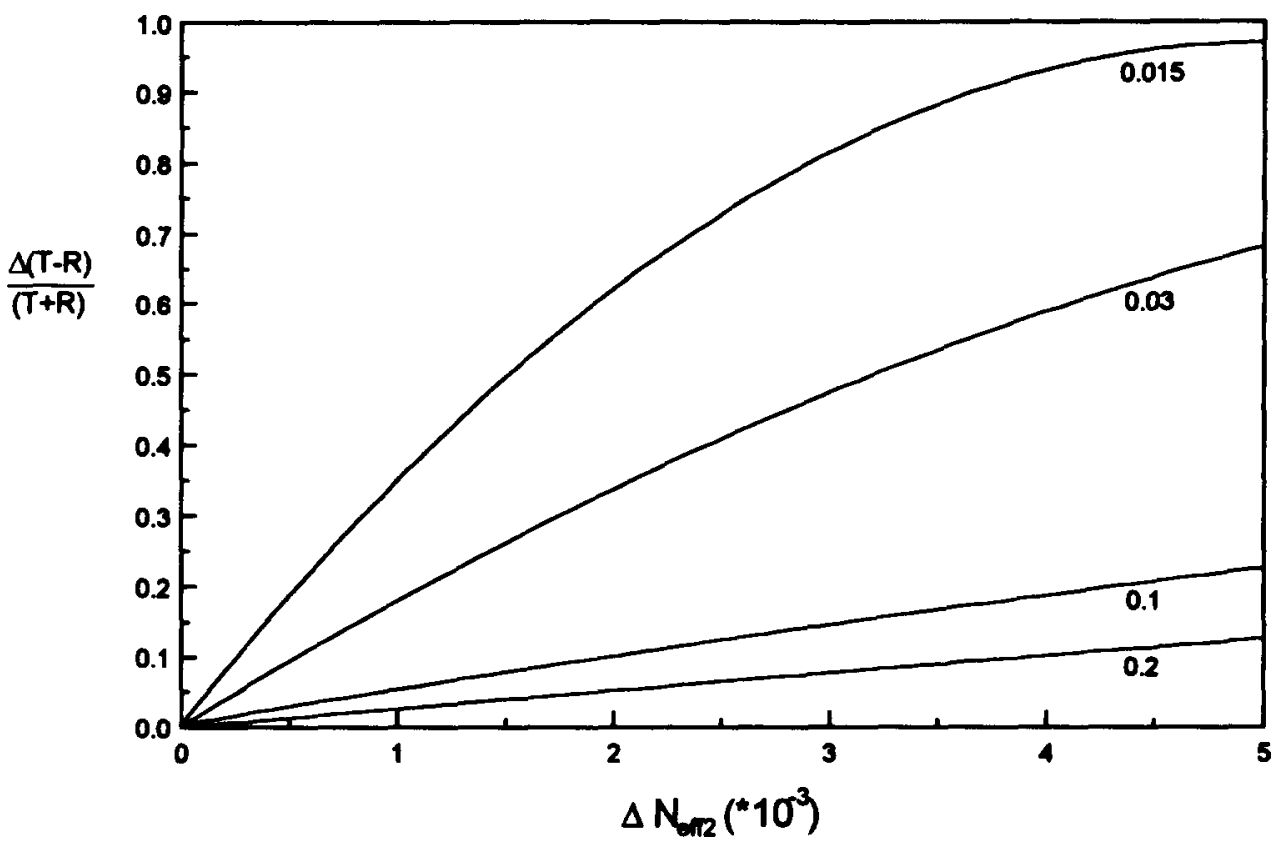

Fig. 4. $\Delta(\mathrm{T}-\mathrm{R})$ for different values of $\boldsymbol{\theta}_{w}(\mathrm{rad})$, as a function of a change in $\mathrm{N}_{\text {eff }}$. Before an adsorption $\mathrm{R}=\mathrm{T}$ and $N_{\text {eff } 2}=1.551$. A $10 \mathrm{~nm}$ average protein layer growth on the waveguide surface corresponds to $\Delta \mathrm{N}_{\text {eff } 2}=5 \times 10^{-3}$. The calculations have been performed for $a \lambda=632.8 \mathrm{~nm}$ TMo mode, a waveguide layer thickness of $130 \mathrm{~nm}$, $\mathrm{n}_{f}=2 \cdot 0, \mathrm{n}_{c}=1 \cdot 34$, and $\mathrm{n}_{c}{ }^{\prime}=1 \cdot 45$. 
complete device, see Fig. 5. The substrate layer is a thermally oxidized $\mathrm{SiO}_{2}$ layer with a thickness of $1.3 \mu \mathrm{m}$ and a refractive index of 1.46 . The high refractive index waveguide layer is a LPCVD (low pressure chemical vapour deposition) $\mathrm{Si}_{3} \mathrm{~N}_{4}$ layer with a refractive index of $2 \cdot 0$, which combines good optical properties (low losses) with good mechanical properties (dense and solid material). In combination with the substrate layer and an aqueous cladding, this waveguide layer provides, at a thickness of $170 \mathrm{~nm}$, a TM $\mathrm{TM}_{0}$ spatial electric field distribution with a good confinement near the waveguide surface. A protective PECVD (plasma enhanced chemical vapour deposition) $1.5 \mu \mathrm{m} \mathrm{SiO}{ }_{2}$ layer with a refractive index of 1.46 is deposited on top of this waveguide surface. This $\mathrm{SiO}_{2}$ layer is partly removed by wet (HF) etching so that a waveguide area results that is unshielded for biochemical reactions. A cleaved and mechanically polished endface of this sensor acts as the end-fire light incoupling part of the device. The outcoupled light is project on two photodiodes fixed on holes previously etched in the waveguide (see Fig. 5b).

\section{Experimental set-up}

An overview of the critical sensor set-up is shown in Fig. 6. A $2.5 \mathrm{~mW} \mathrm{He}-\mathrm{Ne}$ laser beam is chopped and end-fire coupled into the device by a cylindrical lens $(f=10 \mathrm{~mm})$ or a microscope objective $(f=8 \mathrm{~mm})$. At the interface between the shielded and unshielded area of the waveguide, the light splits in the $R$ and $T$ parts. The intensity difference between the $R$ and $T$ parts can be tuned with a rotatable mirror. The intensities of both beams are measured with the two photodiodes fixed on the device. The preamplified signals $T$ and $R$ are both subtracted

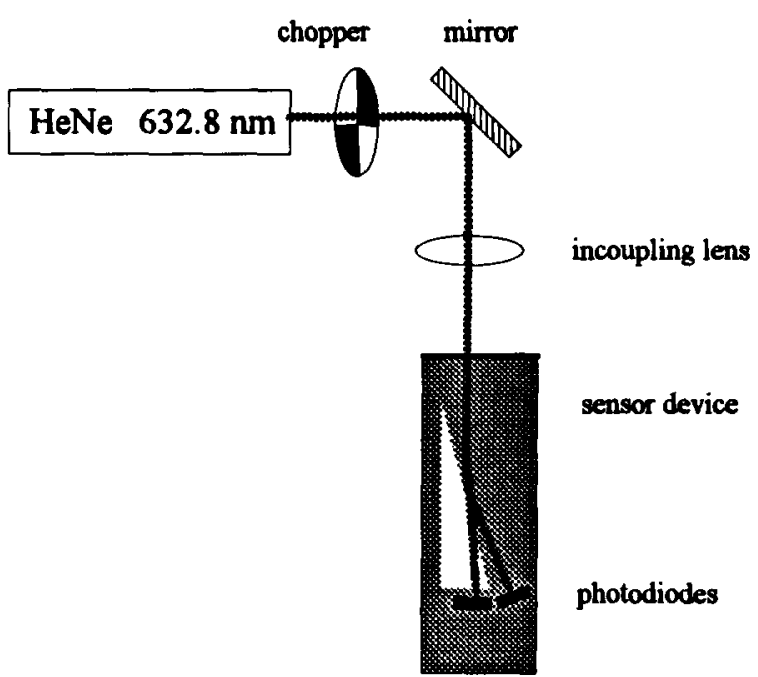

Fig. 6. The experimental set-up.

and added. Both signals are fed into lock-in amplifiers, before being further processed with a computer. The $(R-T)$ is the measurement signal and the $(T+R)$ the normalization signal.

\section{Preparation of protein solutions}

The monoclonal IgG protein anti human chorionic gonadotropin $(\alpha$-hCG, $\quad$ MW $\approx 150 \mathrm{kD})$ and human chorionic gonadotropin (hCG, $\mathrm{MW} \leq 40 \mathrm{kD}$ ) were provided by Organon International (Oss, The Netherlands). Anti-human serum albumin $(\alpha-\mathrm{hSA}, \mathrm{MW} \leq 150 \mathrm{kD})$ and human serum albumin ( $\mathrm{hSA}, \mathrm{MW} \leq 65 \mathrm{kD})$ were bought from Sigma Chemical Co. (St. Louis, MO, USA).

The protein solutions were prepared using a phosphate buffered saline (PBS) solution with $\mathrm{pH}=7 \cdot 35$.

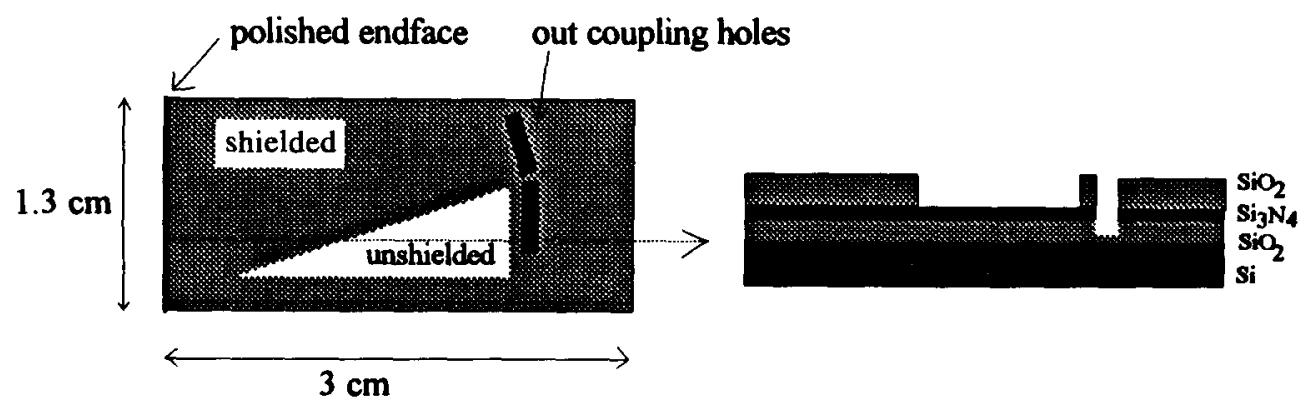

(a)

(b)

Fig. 5. Waveguide design of the critical sensor: (a) top view; (b) cross-section (for details see text). 


\section{RESULTS AND DISCUSSION}

\section{Calibration}

For the given sensor and $n_{m}^{\prime}=1.45$, a layer growth sensitivity of $\mathrm{d} N_{\mathrm{efr} 2} / \mathrm{d} t^{\prime}=$ $4.2 \times 10^{-4} \mathrm{~nm}^{-1}$ has been calculated. The same change to the effective refractive index is made by changing the bulk refractive index of the aqueous medium; for the present sysem: $\mathrm{d} N_{\text {eff } 2} /$ $\mathrm{d} n_{\text {bulk }}=0 \cdot 22$. Glucose solutions with different bulk refractive indices are easy to prepare. The calibration of the sensor is done with these glucose solutions of which the refractive index is measured with an Abbe refractometer. Experiments have been performed with two different types of $\mathrm{TM}_{0}$ waveguide modes. With a $f=8 \mathrm{~mm}$ incoupling objective a highly divergent waveguide mode $\left(\theta_{w} \sim 0.07 \mathrm{rad}\right)$ is created. In another series of experiments a $f=10 \mathrm{~mm}$ cylindrical lens was used so that light only was focused in the horizontal plane. In this case the beam was expected to remain essentially collimated within the waveguide slab $\left(\theta_{w} \sim 0.001 \mathrm{rad}\right)$. However, due to laser beam pointing fluctuations and impurities or roughness scattering within the waveguide structure, the actual beam divergence was higher than expected $\left(\theta_{w} \sim 0.025 \mathrm{rad}\right)$. The results of the measurements can be seen in Fig. 7 . These show that for the $\theta_{w} \sim 0.07 \mathrm{rad}$ mode the experimental results are in reasonable agreement with our model calculations. For the collimated mode $\left(\theta_{w} \sim 0.025 \mathrm{rad}\right)$ the correspondence is much worse. However, it should be realized that

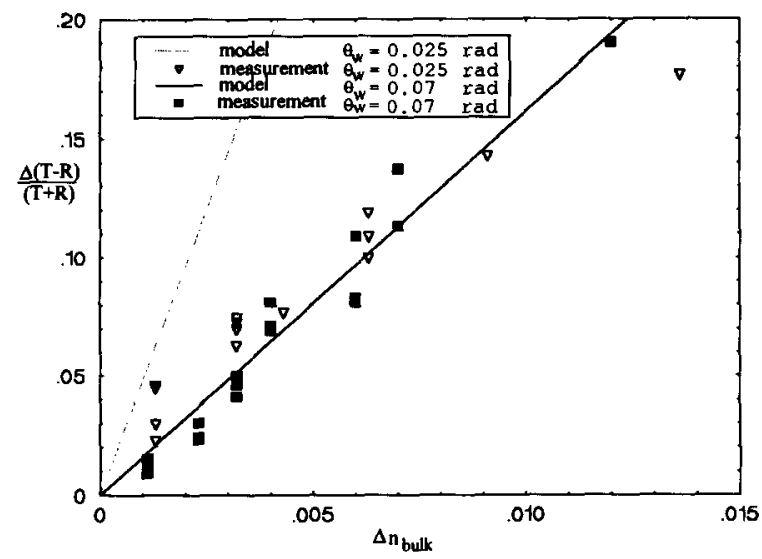

Fig. 7. Calibration of the critical sensor by changing the bulk refractive index with glucose solutions with different concentrations. The same data serve as a test of the model presented in the text. in this situation the experimentally obtained sensitivity is much more dependent on the correct setting of $\theta_{0}$ than for the beam with the low divergence, (equation (11)): the difference in index contrast of the waveguide regions traversed by the $R$ and $T$ beams respectively, results in a difference in attenuation of the two beams. Consequently, the angle at which $R$ and $T$ have equal measured intensities is slightly offset from $\theta_{0}$. This explains the loss in sensitivity. In fact, Fig. 7 indicates that $\theta_{w} \sim 0.07 \mathrm{rad}$ is close to the optimal configuration that can presently be obtained. For this situation a differential sensitivity of $c a .15$ per refractive index unit is obtained, corresponding to a relative intensity change of $3 \times 10^{-2} / \mathrm{nm}$ of layer growth.

In order to test fully the model, experiments with beams of different divergences would be useful. Unfortunately, larger divergences than mentioned above could not be used, in view of the limited width of the employed devices. Smaller divergences were difficult to obtain because of the reasons mentioned above. Also, the uncertainty in $\theta_{0}$ makes a test measurement difficult. Still, we are confident that the employed model is correct: (1) the description given in Fig. 3 provides an obvious explanation; (2) the theoretical line in Fig. 7 calculated from the mathematical quantification of this process was obtained without the use of any fitting parameters; and (3) least square fits to the two sets of measurements in Fig. 7 indeed show a significant trend towards higher sensitivity for smaller divergence.

The important lower layer growth detection limit determining the minimally measurable analyte concentration in immunosensing experiments is determined by the intrinsic sensitivity of the device and the noise during a measurement. For the performed experiments a lower detection limit of $0.02 \mathrm{~nm}$ in average layer growth, or $12 \mathrm{pg} / \mathrm{mm}^{2}$ in analyte coverage, is found. We found that laser beam pointing noise is the main factor determining this limit.

The sensor chips can be reused many times without a measurable loss in sensitivity. In order to reuse the sensors, they are cleaned with a cationic detergent and isopropanol. The sensitivity of a sensor is checked before every new adsorption measurement with a test in which the bulk medium is changed from $\mathrm{H}_{2} \mathrm{O}$ to PBS, with a bulk refractive index difference of $(1 \cdot 8 \pm 0 \cdot 2)$ 
$\times 10^{-3}$. For such a refractive index step we find for a series of 15 independent experiments:

$$
\frac{\Delta(R-T)}{R+T}=0.24 \pm 0.02
$$

\section{Immunosensing experiments with the $\alpha$-HSA/ hSA and $\alpha$-hCG/hCG system}

Prior to the adsorption experiments the waveguide surface is chemically modified with DCDMS (dichlorodimethylsilane) spun on the surface at room temperature. The DCDMS reacts with the waveguide, forming a hydrophobic surface (contact angle $>80^{\circ}$ ). A hydrophobic surface is favourable for obtaining a good physical adsorption in the experiments (Heideman et al., 1994). A hSA or a hCG monolayer is immobolized on this surface by physical adsorption from a concentration of $2 \times 10^{-7} \mathrm{M} \mathrm{hSA}$ or hCG in PBS, as can be seen in Fig. 8 for the hSA. The estimated average thickness of the immobilized hSA layer is ca $2 \pm 1 \mathrm{~nm}$. This thickness has been calculated assuming $n_{m}^{\prime}=1.45$ (Nellen \& Lukosz, 1993), and using the result of the $\mathrm{H}_{2} \mathrm{O}-\mathrm{PBS}$ sensitivity check. After the immobilization of the antigen monolayer a washing step with PBS is performed to remove the antigen molecules present in the bulk solution.

The immunoreaction experiments are performed with the $\alpha$-hSA/hSA and the $\alpha$-hCG/hCG system. The resulting change in the intensity difference is monitored and can be seen for one complete set of $\alpha-\mathrm{hSA} / \mathrm{hSA}$ measurements in

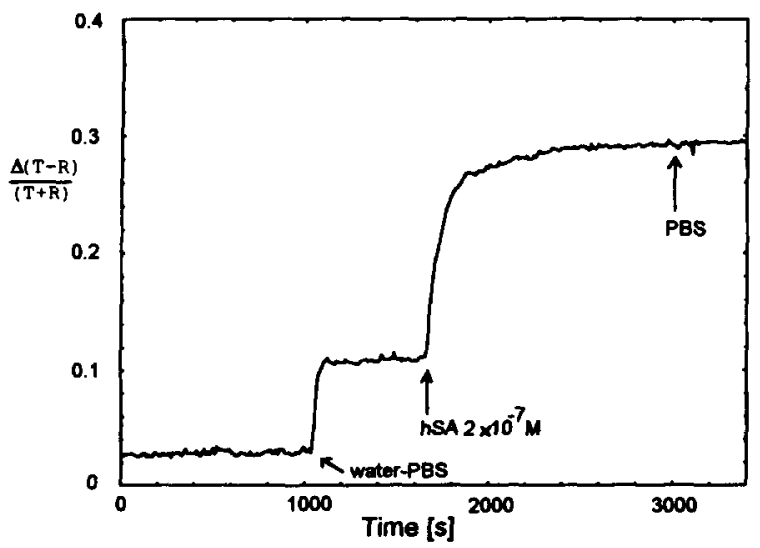

Fig. 8. Immobilization of a hSA monolayer. Addition or washing events have been indicated by arrows.
Fig. 9. In view of the uncontrolled way of sample adding, which was done manually, it was difficult to provide a detailed description of the kinetics of the response. In any case the fast part of, for example, response (f) can never be the result of a temperature difference between the samples, which then should be more than $10^{\circ} \mathrm{C}$. The specificity of the binding is tested by adding a high concentration of different antibodies, for example, $\alpha$-hCG, to the hSA. In all cases the interaction between the corresponding antigen and antibodies proved to be specific. After a complete coverage of the active binding sites on the surface, the measured estimated average layer growth is $5 \pm 1 \mathrm{~nm}$. The obtained results are fitted to an adsorption isotherm describing the binding of molecules to receptor molecules immobilized on a surface:

$$
R+n A \stackrel{k}{\leftrightarrow} R A_{n}
$$

For this equilibrium, the following expression can be deduced (Andrade, 1985):

$$
\Gamma=\frac{n k \cdot[A]^{n}}{1+k \cdot[A]^{n}}
$$

where $\Gamma$ is the fraction of bound receptor molecules, $k$ is the affinity constant of the system, and $n$ denotes the cooperativity in the binding process. When there is a positive cooperativity $(n>1)$, the binding of one molecule helps the binding of the other molecules. The two parameters $n$ and $k$ can be conveniently determined by displaying the results in the form of a Hill plot. This was done in Fig. 10. The plot of $\log (\Gamma / 1-\Gamma)$ versus $\log$ (concentration) should result in a straight line with intercept the values of $\log k$ and slope the cooperativity $n$. From this we find $k \leq 1 \times 10^{-7} \mathrm{M}^{-1}$ for the $\alpha-\mathrm{hSA} / \mathrm{hSA}$, and $k \leq 5 \times 10^{-7} \mathrm{M}^{-1}$ for the $\alpha$-hCG/hCG system, which is in line with previous results (Heideman et al., 1994). For both systems a cooperativity $n \leq 1$ is found.

\section{CONCLUSIONS}

The model describing the sensitivity of the critical sensor corresponds with the measurements when a high divergence waveguide mode is used. The measurements performed with the low divergence waveguide mode do not correspond, and due to laser beam pointing fluctuation, and impurity or 


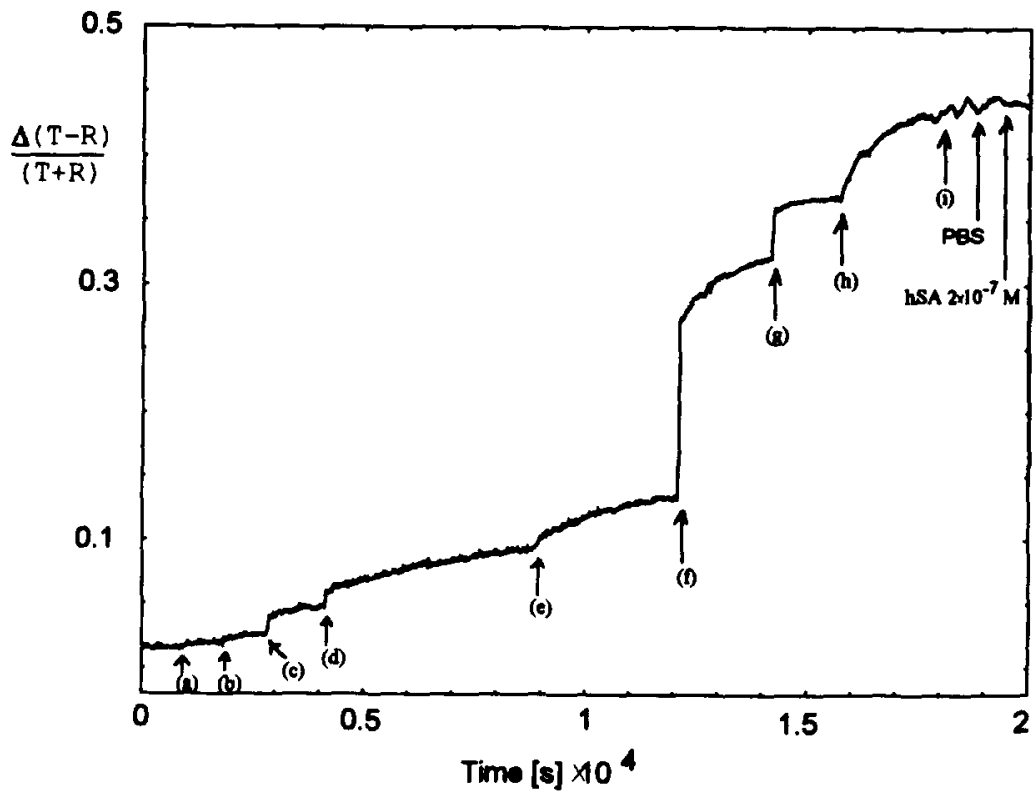

Fig. 9. Immunoreaction experiments. Different concentrations of $\alpha-h S A$ in PBS solution are added: (a) $1 \times 10^{-9} M$, (b) $4 \times 10^{-9} \mathrm{M}$, (c) $1 \times 10^{-8} \mathrm{M}$, (d) $2 \times 10^{-8} \mathrm{M}$, (e) $4 \times 10^{-8} \mathrm{M}$, (f) $1.5 \times 10^{-7} \mathrm{M}$, (g) $2.5 \times 10^{-7} \mathrm{M}$, (h) $4.7 \times 10^{-7} \mathrm{M}$, (i) $8 \times 10^{-7} \mathrm{M}$.

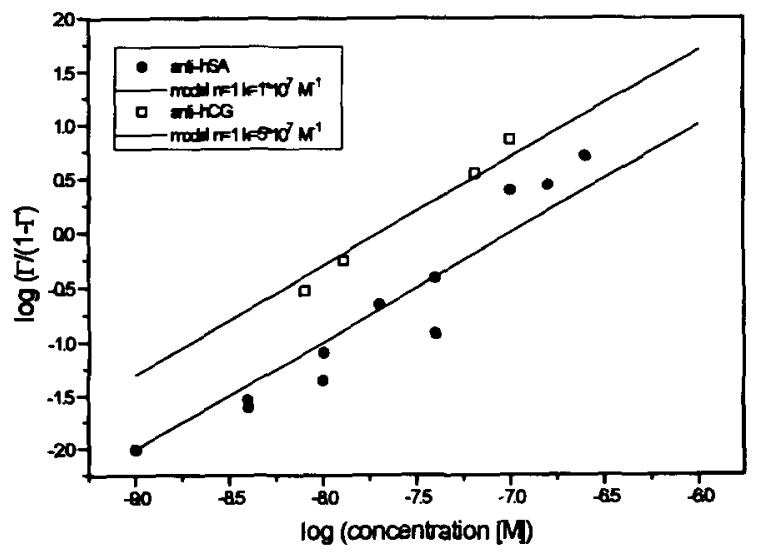

Fig. 10. Afffinity and cooperativity measurement. Hill plot of $\alpha-h S A / h S A$ and the $\alpha-h C G / h C G$ immunosensing results: $-\alpha-h C G, \cdots \alpha-h S A$.

roughness scattering, the obtained sensitivity is not significantly better than that found for the high divergence mode.

During the experiments the sensors were reused many times without a measurable decrease in sensitivity.

Immunosensing experiments performed with the $\alpha$-hSA/hSA and the $\alpha$-hCG/hCG confirm the possible use of the "critical" sensor as a simple immunosensor device. The lower detection limit obtained is $0.02 \mathrm{~nm}$ in layer growth, or $12 \mathrm{pg} / \mathrm{mm}^{2}$ in analyte surface coverage. This is comparable to the performance of the surface plasmon resonance sensors (Kooyman et al., 1991). However, the advantages of the "critical" sensor in comparison with the surface plasmon resonance sensors are the simple fabrication and the simple operation of the sensor, and the fact that we no longer need a thin gold layer. In comparison with the Mach-Zehnder interferometer sensor, the sensor is less sensitive (Schipper et al., 1994), but also less complex in fabrication and adjustment prior to a measurement. Thus, for applications which do not need an extremely high sensitivity, for example, in indirect immunosensing experiments, the use of the "critical" sensor is more suitable. For example, the present performance of the "critical" sensor is adequate for pesticide competitive binding, such as was demonstrated recently (Bier \& Schmid, 1994).

Another point, not exclusively pertaining to the device described in the present paper, is the common difficulty of label-free evanescent wave methods to discriminate signals originating from specific or non-specific surface interactions. To solve this, we have to await the next generation of immunosensors that employ a multisensing detection scheme accompanied by a pattern recognition algorithm. 


\section{ACKNOWLEDGEMENT}

These investigations have been supported by the Dutch Technology Foundation (STW).

\section{REFERENCES}

Andrade, J.D. (1985). Principles of protein adsorption. In: Surface and Interfacial Aspects of Biomedical Polymers, Vol. 2, J.D. Andrade (ed.), Plenum Press, New York.

Bier, F.F. \& Schmid, R.D. (1994). Real time analyses of competitive binding using the grating coupler immunosensors for pesticide detection, Biosensors \& Bioelectronics, 9, 125-130.

Cush, R., Cronin, J.M., Stewart, W.J., Maule, C.H., Molloy, J. \& Goddard, N.J. (1993). The resonant mirror: a novel optical biosensor for direct sensing of biomolecular interactions. Part 1: Principle of operation and associated instrumentation. Biosensors \& Bioelectronics, 8, 347-353.

Heideman, R.G., Kooyman, R.P.H. \& Greve, J. (1993). Performance of a highly optical waveguide Mach-Zehnder interferometer immunosensor. Sensors \& Actuators B, 10, 209-217.

Heideman, R.G., Kooyman, R.P.H. \& Greve, J. (1994). Immunoreactivity of adsorbed anti human chorionic gonadotropin studied with an optical waveguide interferometric sensor. Biosensors \& Bioelectronics, 9, 33-43.

Kooyman, R.P.H. \& Lechuga, L.M. (1995). Evanescent wave immunosensors. CRC Biosensors Handbook. CRC Press, Boca Raton, FL (in press).

Kooyman, R.P.H., Lenferink, A.T.M., Eenink, R.G. \& Greve, J. (1991). Vibrating mirror surface plasmon resonance immunosensor. Anal. Chem., 63, 83-85.

Liedberg, B., Lundström, I. \& Stenberg, E. (1993). Principles of biosensing with an extended coupling matrix and surface plasmon resonance. Sensors \& Actuators B, 11, 63.

Lukosz, W. (1988). Sensitivities of integrated optical grating and prism couplers as (bio) chemical sensors. Sensors \& Actuators, 15, 273-284.

Lukosz, W. (1991). Principles and sensitivities of integrated optical and surface plasmon sensors for direct affinity sensing and immunosensing. Biosensors \& Bioelectronics, 6, 215-225.

Nellen, P.M. \& Lukosz, W. (1993). Integrated optical input grating couplers as direct affinity sensors. Biosensors \& Bioelectronics, 8, 129-147.

Schipper, E.F., Kooyman, R.P.H. \& Greve, J. (1995). Feasibility of optical waveguide immunosensors for pesticide detection: Physical aspects. Sensors \& Actuators B, 24-25, 90-93. 